\title{
A Randomized Controlled Effectiveness Trial for PSA Screening Decision Support Interventions in Two Primary Care Settings
}

\author{
Carmen L. Lewis, M.D., M.P.H. ', Jared Adams, M.D., Ph.D. ${ }^{3,4}$, Ming Tai-Seale, Ph.D. ${ }^{3}$, \\ Qiwen Huang, MS ${ }^{3}$, Sarah B. Knowles, Ph.D., M.P.H. ${ }^{3}$, Matthew E. Nielsen, M.D. ${ }^{5}$, \\ Michael P. Pignone, M.D., M.P.H., 10 , Louise C. Walter, M.D. ${ }^{6,7}$, and Dominick L. Frosch, PhD $D^{3,8,9}$
}

\begin{abstract}
'Division of General Internal Medicine, Department of Medicine, University of Colorado School of Medicine, Aurora, CO, USA; ${ }^{2}$ Division of General Internal Medicine and Clinical Epidemiology, Department of Medicine, University of North Carolina at Chapel Hill, Chapel Hill, NC, USA; ${ }^{3}$ Palo Alto Medical Foundation Research Institute, Palo Alto, CA, USA; ${ }^{4}$ UCSF Philip R. Lee Institute for Health Policy Studies, San Francisco, CA, USA;

${ }^{5}$ Department of Urology, University of North Carolina at Chapel Hill, Chapel Hill, NC, USA; ' UCSF Division of Geriatrics, San Francisco, CA, USA; 7 San Francisco Veterans Affairs Medical Center, San Francisco, CA, USA; ${ }^{8}$ Gordon and Betty Moore Foundation, Palo Alto, CA, USA; ${ }^{9}$ Division of General Internal Medicine \& Health Services Research, Department of Medicine, UCLA, Los Angeles, CA, USA; ${ }^{10}$ Cecil G. Sheps Center for Health Services Research, University of North Carolina at Chapel Hill, Chapel Hill, NC, USA.
\end{abstract}

BACKGROUND: Decision support interventions (DESIs) provide a mechanism to translate comparative effectiveness research results into clinical care so that patients are able to make informed decisions. Patient decision support interventions for prostate-specific antigen (PSA) have been shown to promote informed decision making and reduce PSA testing in efficacy trials, but their impact in real world settings is not clear.

OBJECTIVE: We performed an effectiveness trial of PSA decision support interventions in primary care.

DESIGN: A randomized controlled trial of three distribution strategies was compared to a control.

PARTICIPANTS: Participants included 2,550 men eligible for PSA testing (76.6 \% of the eligible population) and 2001 survey respondents (60.1\% survey response rate).

INTERVENTIONS: The intervention groups were: 1) mailed the DESI in DVD format, 2) offered a shared medical appointment (SMA) to view the DESI with other men and discuss, and 3) both options.

MAIN MEASURES: We measured PSA testing identified via electronic medical record at 12 months and DESI use by self-report 4 months after the intervention mailing.

KEY RESULTS: We found no differences in PSA testing across the three distribution strategies over a year-long follow-up period: $21 \%, 24 \%, 22 \%$ in the DESI, SMA, and combined group respectively, compared to $21 \%$ in the control group $(p=0.51)$. Self-reported DESI use was low across all strategies at 4 months: $16 \%$ in the mailed DESI group, $6 \%$ in the SMA group, and $15 \%$ in the combined group $(p=<0.0001)$.

CONCLUSIONS: Mailing PSA decision support interventions or inviting men to shared medical appointments unrelated to a primary care office visit do not appear to promote informed decision making, or change PSA testing behavior.

Trial Registration: ClinicalTrials.gov Identifier: NCTO1241656

Received August 20, 2014

Revised January 12, 2015

Accepted January 23, 2015

Published online February 10, 2015
KEY WORDS: cancer prevention; prostate cancer; medical decision making; prostate-specific antigen; early detection of cancer.

J Gen Intern Med 30(6):810-6

DOI: $10.1007 / \mathrm{s} 11606-015-3214-9$

(C) Society of General Internal Medicine 2015

$\mathrm{D}$ ecision support interventions (DESIs) provide a mechanism to translate comparative effectiveness research results into clinical care, so that patients are able to make informed decisions. In efficacy trials, DESIs have been shown to increase knowledge, activate patients to participate in shared decision making, resulting in decisions that are more informed and consistent with patient values. ${ }^{1}$ These tools have been developed for many medical decisions; however, prostate cancer screening with prostate specific antigen (PSA) is a decision where informed decision making has been strongly endorsed. ${ }^{2-5}$

Patient DESIs for PSA screening have been shown to promote informed decision making and reduce prostatespecific antigen (PSA) screening in efficacy trials. ${ }^{6,7}$ However, the evidence supporting the use of decision support comes primarily from randomized controlled trials performed in ideal conditions - ensuring exposure to the intervention-with select populations of research volunteers. Several wellconducted studies of these PSA-DESIs are available but have been limited, either because participation required active consent, ${ }^{8}$ limiting generalizability, or the studies were performed at single sites and not randomized. ${ }^{9,10}$ Several other studies have explored using PSA DESIs in conjunction with counseling or shared medical appointments, but these have also been limited by select samples. ${ }^{11,12}$ Therefore, little is known about the effectiveness of PSA-DESIs in an unselected population of men in "real world" primary care settings, despite recommendations that they be used to facilitate decisions about PSA screening. 
To address this gap, we conducted an effectiveness trial to determine the impact of several distribution methods in two primary care settings. We designed the trial to maximize reach and generalizability, to mimic conditions in primary care where distribution of PSA-DESIs has been recommended. Specifically, we conducted a randomized controlled trial comparing three distribution methods (mailing the DESI, offering a shared medical appointment for DESI viewing, or both options) to a control group. We hypothesized that providing men with access to PSA-DESIs would result in uptake of the DESIs by men in the intervention groups and a decrease in PSA screening, as has been found in some efficacy trials. ${ }^{1,7}$ We also hypothesized that the use of DESIs would differ by distribution strategy; specifically, we hypothesized men offered both options would be more likely to use the DESIs.

\section{METHODS}

\section{Setting}

The trial was conducted in two geographically diverse primary care sites. Site one was in Northern California, with seven primary care practices, and site two was an academic general internal medicine practice in the southeast United States.

\section{Study Population and Randomization}

The study received approval from the Institutional Review Boards of each institution. To assure that the sample reflected men in primary care practices, we obtained a HIPAA waiver to identify eligible men using the electronic health record and to analyze their PSA testing information from their medical record unless they opted out of participation. Men were eligible for the study if they were (1) aged 50-75 years, (2) did not have a prostate cancer diagnosis, (3) had not had a PSA test in the past 10 months and, (4) had not seen their primary care physician in the last 3 months.

\section{Interventions}

Approximately every 2 weeks during the intervention period (February 2011 to December 2012), a list of men who met the eligibility criteria was randomly selected from the pool of eligible patients in the electronic medical records (EMRs). Patients were mailed a letter describing the study and a postage-paid opt-out card. The mailing included information about why PSA testing is controversial, and recommendations by professional organizations indicating that men should be informed and decide whether they want to be tested. In addition to the letter, depending on their randomization group, men also received: (1) a DESI in DVD format; (2) an invitation to participate in a shared (group) medical appointment (SMA) to watch and discuss the DESI with a mid-level healthcare provider and other patients; (3) both the DVD DESI and an invitation to participate in a SMA or (4) no additional intervention material. The PSA-DESI is 31 minutes long and was developed by the Informed Medical Decisions Foundation. In several controlled trials, the program has been found to be balanced, increase knowledge and decrease desire for PSA testing.$^{12,13}$ The content describes tests to detect prostate cancer, options for treatment if cancer is found, with information about the associated benefits and risks, and that the mortality benefit of treatment is uncertain.

\section{Participant Survey 4 Months after Study Mailing}

We surveyed men 4 months after the study materials were mailed. We first sent a written survey to men who had not opted out of the study, and if the survey was not returned within 2 weeks, research staff called men to complete the survey on the phone. Men not contacted within 8-10 weeks were considered lost to follow-up but were still analyzed for the PSA testing outcome, unless they had opted out of the study.

\section{Outcomes}

The primary outcome was PSA screening at 12 months postmailing. PSA tests were identified from EMR data extraction.

Secondary outcomes determined by self-report during the survey included DESI viewing and contact with providers about PSA testing, PSA decision-specific knowledge, ${ }^{14}$ and attitudes toward PSA testing.

\section{Statistical Analyses}

Statistical analyses consisted of both the intention-to-treat and a priori secondary analyses. We used $\chi^{2}$ tests to compare the group differences in PSA screening. Associations between the primary outcome and categorical variables were tested using Pearson's $\chi^{2}$ and continuous variables were tested using Student's t-test. Secondary a priori analyses were conducted on men who viewed the DESI. In these analyses, survey non-respondents were classified as having not viewed the DESI.

Covariates thought to be associated with PSA screening had been selected based on prior work ${ }^{12,13,15}$ and clinical experience, and included race/ ethnicity (African American, Latino/ Hispanic, White, Asian/Pacific Islander, other), age, sex, marital status, education (less than high school, high school/ GED, some college, college graduate), income, insurance, geographic site (site 1 or 2), and prior PSA screening (as captured from the electronic medical record within 1 year prior to enrollment). Covariates with a significance level ( $P$ value) less than 0.05 were included in the multivariate logistic regression model. Covariates for a logistic regression model for DESI viewing were selected in the same manner. For both outcomes (PSA screening and DESI viewing), the resulting multivariate logistic regression that was used to estimate odd ratios and $95 \%$ confidence intervals $(95 \% \mathrm{CI})$, controlling for group, study site, prior PSA screening, and insurance. (Please see the Appendix for additional statistical details.) 
All analyses were executed using a two-sided $\alpha=0.05$ and all analyses used SAS software version 9.2. We based our sample size calculation on the primary outcome, prostate cancer screening. Because we planned to perform pairwise comparisons using analysis of variance between the four intervention groups, we used a Bonferroni correction of 6 (effective two-sided alpha of 0.05 / $6=0.0083$ ) in our sample size calculation. We assumed that the control group would have a screening rate of $55 \%$ based on Behavioral Risk Factor Surveillance System (BRFSS) screening rates at the time of the proposal. ${ }^{16}$ We estimated that 650 men in each group would provide $80 \%$ power to detect a $10 \%$ difference in screening test completion.

\section{RESULTS}

In all, 4,028 men were randomly selected from the electronic medical records, 701 men were ineligible and 777 men opted out of the study (Fig. 1). Of the 2,550 participants who remained eligible and did not opt out, 2001 completed a questionnaire by mail or phone. This represents a $76.6 \%$ participation rate of eligible participants and a $60.1 \%$ response rate for the survey.

\section{Baseline Characteristics}

Baseline characteristics were comparable across study groups (Table 1). There were significant differences between the two settings in terms of race/ethnicity, education, and income, reflecting expected differences in the demographics of the two primary care populations.

PSA Testing. None of the distribution strategies demonstrated a significant difference in PSA testing (Table 2): $21 \%, 24 \%$, and $22 \%$ in the DESI, SMA, and combined groups, respectively, compared to $21 \%$ in the control group ( $p=0.51$ ).

We found differences in PSA testing by site, but not across the intervention groups at each site. For site one, the proportion receiving PSA testing was $34 \%, 37 \%, 34 \%$ in the DESI, SMA, and combined groups, respectively, compared to $32 \%$ in the control group ( $p=0.66$ ); for the second site, the proportions were $9 \%$ in the DESI group, $12 \%$ in the SMA group, $10 \%$ in the combined group, and $10 \%$ in the control group $(p=0.57)$.

When we examined factors associated with PSA testing in multivariate logistic regression model across the full sample, we found that site, prior history of testing, and insurance were significant. A participant from site 1 had 3.0 times the odds of having a PSA test during the 12-month follow-up as a participant at the second site (95\% CI: 2.3-3.8). Men who had a prior history of PSA testing had 4.1 times the adjusted odds of having a PSA test, compared to those who were screening naïve (95 \% CI: 3.1-5.4). Men who had Medicare or Medicaid, or HMO, or PPO/FFS had 3.6 (95\% CI: 1.8-7.4), 3.0 (95 \% CI: 1.5-6.3), and 3.7 times (95 \% CI: 1.8-7.5), respectively, the adjusted odds of having a PSA test, compared to those without insurance.

DESI Viewing. Among the three distribution strategies, DESI viewing was low (Table 2). The combined strategy of offering an invitation to a SMA in addition to a mailed DESI did not lead to increased DESI viewing among participants (16\% in DESI group vs. $15 \%$ in the combined DESI+SMA group, $p=0.79)$. Further, few men attended the SMA groups [5.0 \% $(33 / 656)$ in the SMA group and $3.6 \%(23 / 636)$ in the combined group].

Contact with Provider. At 4 months, 37 \% (741/2,001) of the cohort reported a primary care visit. There were no significant differences between intervention groups for likelihood of having a PCP visit [40 \% DESI group (194/485)], $36 \%$ SMA group (185/510), $37 \%$ combined group (180/485) vs. $37 \%$ control group (182/489); $p=0.65)$. Among men who reported a visit, we found no significant differences in the proportion of men who reported discussions with the doctor about PSA testing during the visit [DESI $44 \%(85 / 192)$, SMA $35 \%(64 / 184)$, combined $37 \%$ (66/177) vs. control $41 \%(74 /$ $182) ; p=0.26]$. Additionally, few men in all groups attempted to contact the doctor about PSA screening outside of a visit (6\%), and there were no differences by intervention group (DESI $7 \%$, SMA $8 \%$, combined $7 \%$ vs. control $5 \%$; $p=0.45)$. Finally, among those who had a provider visit, there was no difference in PSA testing [DESI $29 \%(56 / 194)$, SMA $32 \%(60 / 185)$, combined $31 \%(55 / 180)$ vs. control group $26 \%(48 / 182 ; p=0.63)]$.

DESI Efficacy. Compared to controls, DESI viewers were significantly more likely to have a PSA test at 12 months [DESI viewers $30 \%(55 / 186)$ vs. the control group $21 \%$ $(131 / 627) ; p=0.01]$. Among men who reported an office visit, DESI viewers were more likely than controls to engage in discussions about PSA screening with their doctors [DESI viewers $57 \%(42 / 74)$ vs. control group $41 \%(74 / 182)$; $p=0.02]$.

DESI viewing was associated with higher knowledge scores (DESI viewers $76 \% \pm 24 \%$ vs. $60 \% \pm 28 \%$; $p<0.001$ ) and was also associated with less positive attitudes around routine PSA screening for men their age [DESI viewers $54 \%$ $(99 / 183)$ vs. control $65 \%(323 / 495) ; p=0.008]$.

\section{DISCUSSION}

In this effectiveness trial, we found no differences in the effect of DESI distribution strategies on PSA testing. Use of the interventions across the intervention groups was low, ranging from 6 to $16 \%$. Contrary to our hypothesis, the combined SMA-DESI group did not result in higher DESI uptake compared to the other two intervention groups. 


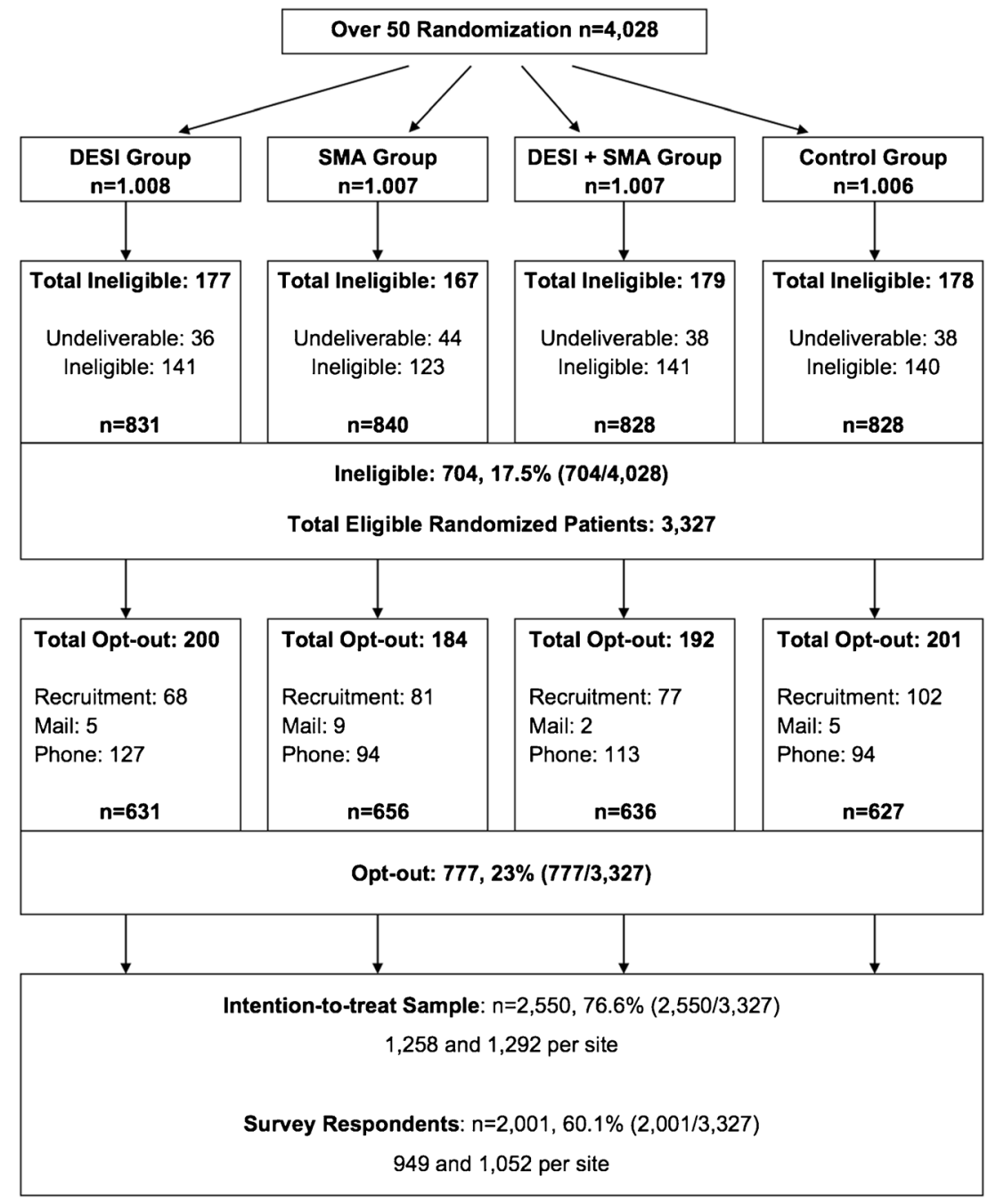

Figure 1 Randomization, eligibility, and participation

Other studies have mailed DVDs, ${ }^{8,9,17,18}$ and some have also encouraged group discussions regarding PSA screening. ${ }^{11,12}$ However, our study is larger than these previous studies and was performed in a more generalizable population in two settings in the US. Unlike our current findings, a much smaller previous study by Frosch et al. ${ }^{12}$ demonstrated that a group assigned to discussion (similar to our SMA) plus DESI was less likely to have a PSA test completed compared to usual care. However, this population was selected from an ongoing screening program, and therefore does not likely reflect a general primary care population. Similar to our findings, Taylor and colleagues found no differences in PSA testing by self-report at 13 months as a secondary outcome in a large efficacy trial comparing web-based decision aid to mailed print PSA DESIs. ${ }^{8}$ In Taylor et al.'s study, informed consent was required, and the authors reported a participation rate of less than $40 \%$. In our study, the participation rate was over $75 \%$ and the design allowed us to review PSA testing via EMR for those who did not opt out.

Despite the fact that informed decision making has been recommended regarding PSA testing by multiple professional organizations, ${ }^{5,19,20}$ we found minimal impact of the intervention despite the high reach of the mail out approach, primarily because of the low uptake of the of DESIs. This low uptake occurred despite significant media attention when the US Preventive Services Task Force released new PSA recommendations during the trial, ${ }^{21}$ where the media attention may have increased interest in men learning more about PSA testing. However, we found no difference in uptake before and after the guidelines were released. The low use of DESIs is consistent with previous non-randomized effectiveness studies that mailed out colorectal cancer screening and PSA DVDs, where uptake ranged from 10 to $36 \% .^{9,17,18}$ Given the rigorous design and the coincidental timing of our study with significant media attention, mailing PSA-DESIs or using SMAs unrelated to an upcoming visit do not appear to be effective methods to promote informed decision making for PSA testing. However, in a smaller study comparing an educational pamphlet to a DVD, researchers have demonstrated uptake of greater than $50 \%$ when mailings of these materials were tied to an upcoming office visit. ${ }^{22}$ So, uptake may have been higher for this study if the mailings were tied to an upcoming 
Table 1 Baseline Participant Characteristics, by Site and Intervention Group

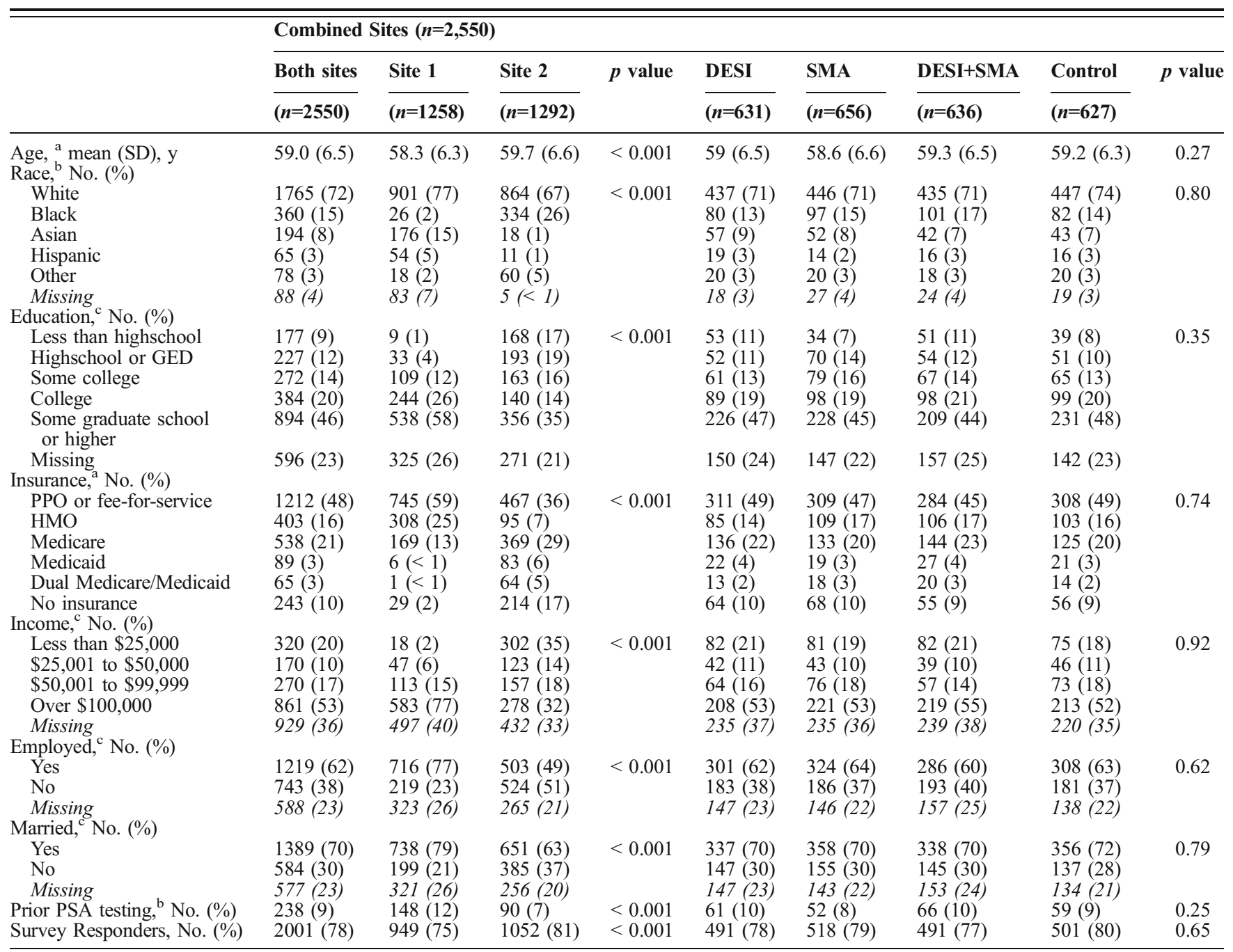

${ }^{a}$ Data source: Electronic Medical Record

${ }^{b}$ Data source: Electronic Medical Record and Patient Surveys

${ }^{c}$ Data source: Patient Surveys

office visit, but this approach would introduce a selection bias, which our current design sought to avoid.

There are several other potential explanations for the low uptake. First, we did not engage providers to promote DESI use or train them in informed/shared decision making. Recently, DESIs have been developed to engage patients during the office visit, ${ }^{23}$ which could potentially be more effective in

Table 2 Primary and secondary outcomes data by intervention group

\begin{tabular}{lllll}
\hline \hline & DESI & SMA & DESI+SMA & Control \\
\hline $\begin{array}{l}\text { PSA testing at 12 } \\
\text { months, No. (\%) }\end{array}$ & $134(21)$ & $158(24)$ & $139(22)$ & $131(21)$ \\
$\begin{array}{l}\text { DESI viewing at 4 } \\
\text { months, No. (\%) }\end{array}$ & $78(16)$ & $33(6)$ & $75(15)$ & N/A \\
\hline
\end{tabular}

${ }^{a}$ Whole baseline sample: DESI $n=631$, SMA $n=656, D E S I+S M A n=$ 636, Control $n=627$

${ }^{b}$ Sample among participants who completed the follow-up survey: DESI $n=491, S M A n=518, D E S I+S M A n=491$ increasing uptake. However, a significant downside to this approach is relying on providers to bring up the topic, rather than having a systematic approach. Further, competing priorities may limit the reach of this approach, and thus decrease its overall impact. ${ }^{24}$ Additionally, the letter sent with the intervention was not endorsed by the providers, and this may have also decreased the likelihood of patients using the DESI.

In contrast to some efficacy trials that demonstrated a decrease in PSA use with DESI, we actually found that participants who reported using the DESI were more likely to obtain a PSA test compared with controls, despite having higher knowledge scores and a less positive impression of PSA screening. The inconsistency of our findings with many efficacy trials ${ }^{6,7}$ where DESI viewing is assured, suggests that participants in efficacy trials who consent to participate may be different than men in our effectiveness study, which did not require opt-in consent. It also suggests that those who elected to use the DESI may differ from those who chose not to use it. The self-selected men who used the DESI may be more 
engaged with PSA testing and have higher baseline knowledge. Our results suggest effectiveness trials are important to determine the potential uptake and impact of DESIs in more generalizable samples before recommendations are made to encourage them as a standard of care in clinical practice. ${ }^{25}$

Our study has several important limitations. First, we relied on men to contact their providers and set up a visit. Although at 4 months, a significant proportion had been seen, our results may have been different if we had tied mailing of DESIs to a scheduled visit. Next, although our study was designed to maximize generalizability, men who opted out of the study or did not respond to the survey may be different than those who did participate. However, it is important to note that a strength of the study was that our study design allowed us to determine PSA testing for the survey non-respondents who did not opt-out of study participation. We have found in previous work that men who do not respond to surveys are very unlikely to have used decision support interventions. ${ }^{17}$ Finally, although we had a high participation rate $(76 \%)$ and survey response rate $(60 \%)$, the proportion of men who chose to use the DESI was small and self-selected. Our results demonstrate that effectiveness trials can be useful to determine uptake of DESIs in real world practice, as effects identified in highly controlled efficacy trials may not replicate in larger effectiveness trials. Consequently, both efficacy and effectiveness studies are needed to determine the impact of DESIs in clinical practice.

In conclusion, we found no differences in the impact of DESI distribution strategies on PSA testing 12 months after we mailed study materials to men eligible for PSA testing. Use of the interventions across the intervention groups was low. Mailing PSA decision support interventions or inviting men to shared medical appointments unrelated to a primary care office visit does not appear to promote informed decision making for PSA testing or change PSA testing behavior.

Acknowledgements: Contributors: Isheen Herrera, BS; Parisa Montazeri, BS; Katie Stanley, BA; and Will Bassett, BS, were employed as study staff at site 1 and were involved in the data collection and data management for the trial. Lisa Werner, MA Ed, and Chris DeLeon, $B A$, were employed as study staff at site 2 and each was involved in the data collection and data management for the trial.

Funders: The study was supported by a grant from the U.S. Department of Health and Human Services (DHHS), Assistant Secretary for Planning and Evaluation (R18 AE000023). Dr. Walter also received funding from the National Institute on Aging (K24 AG041180), and the National Cancer Institute (RO1 CA1334425-01). Prior presentations: Presented in part at The International Shared Decision Making Conference, 18 June 2013, Lima, Peru

Conflict of Interest: Authors Lewis, Adams, Pignone, and Frosch have received previous grant funding from the Informed Medical Decisions Foundation, which developed the PSA DESI implemented in the study. Dr Pignone serves as a medical editor of the Informed Medical Decisions Foundation.

Corresponding Author: Carmen L. Lewis, M.D., M.P.H.; Division of General Internal Medicine, Department of Medicine, University of Colorado School of Medicine Mail stop B180, Academic Office 1, Room 8415, $12631 \mathrm{E} 17$ th Ave Aurora CO 80045 USA ;(e-mail: Carmen.L.Lewis@ucdenver.edu).

\section{REFERENCES}

1. Stacey D, Bennett CL, Barry MJ, Col NF, Eden KB, Holmes-Rovner M, et al. Decision aids for people facing health treatment or screening decisions. Cochrane Database Syst Rev. 2011;10, CD001431.

2. Centers for Disease Control and Prevention. http://www.cdc.gov/cancer/ prostate/basic info/screening.htm. Accessed Jan 232015.

3. National Cancer Institute FactSheet: Prostate-Specific Antigen (PSA) Test. National Cancer Instititute. 2012. http://www.cancer.gov/cancertopics/ factsheet/detection/PSA. Accessed Jan 232015.

4. American Cancer Society recommendations for prostate cancer early detection. American Cancer Society. 2012. http://www.cancer.org/cancer/prostatecancer/moreinformation/prostatecancerearlydetection/prostate-cancer-early-detection-acs-recommendations. Accessed Jan 232015.

5. Carter HB, Albertsen PC, Barry MJ, Etzioni R, Freedland SJ, Greene KL, et al. Early Detection of Prostate Cancer: AUA Guideline. J Urol. 2013;190(2):419-26.

6. Volk RJ, Hawley ST, Kneuper S, Holden EW, Stroud LA, Cooper CP, et al. Trials of decision aids for prostate cancer screening: a systematic review. Am J Prev Med. 2007;33(5):428-34.

7. Evans R, Edwards A, Brett J, Bradburn M, Watson E, Austoker J, et al. Reduction in uptake of PSA tests following decision aids: systematic review of current aids and their evaluations. Patient Educ Counsel. 2005;58(1): 13-26.

8. Taylor KL, Williams RM, Davis K, Luta G, Penek S, Barry S, et al. Decision Making in Prostate Cancer Screening Using Decision Aids vs Usual Care: A Randomized Clinical Trial. JAMA internal medicine. 2013.

9. Brackett C, Kearing S, Cochran N, Tosteson AN, Blair BW. Strategies for distributing cancer screening decision aids in primary care. Patient Educ Counsel. 2010;78(2):166-8.

10. Miller KM, Brenner A, Griffith JM, Pignone MP, Lewis CL. Promoting decision aid use in primary care using a staff member for delivery. Patient Educ Counsel. 2012;86(2):189-94.

11. Driscoll DL, Rupert DJ, Golin CE, McCormack LA, Sheridan SL, Welch BM, et al. Promoting prostate-specific antigen informed decision-making. Evaluating two community-level interventions. Am J Prev Med. 2008;35(2):87-94.

12. Frosch DL, Kaplan RM, Felitti V. The evaluation of two methods to facilitate shared decision making for men considering the prostate-specific antigen test. J Gen Intern Med. 2001;16(6):391-8.

13. Frosch DL, Kaplan RM, Felitti VJ. A randomized controlled trial comparing internet and video to facilitate patient education for men considering the prostate specific antigen test. J Gen Intern Med. 2003;18(10):781-7.

14. Radosevich DM, Partin MR, Nugent S, Nelson D, Flood AB, Holtzman $\mathbf{J}$, et al. Measuring patient knowledge of the risks and benefits of prostate cancer screening. Patient Educ Counsel. 2004;54(2):143-52.

15. Frosch DL, Bhatnagar V, Tally S, Hamori CJ, Kaplan RM. Internet patient decision support: a randomized controlled trial comparing alternative approaches for men considering prostate cancer screening. Arch Intern Med. 2008;168(4):363-9.

16. Brawley OW, Ankerst DP, Thompson IM. Screening for prostate cancer. CA: a cancer journal for clinicians. 2009;59(4):264-73.

17. Lewis CL, Brenner AT, Griffith JM, Pignone MP. The uptake and effect of a mailed multi-modal colon cancer screening intervention: a pilot controlled trial. Implementation science. 2008;3:32.

18. Lewis CL, Pignone MP. Promoting informed decision-making in a primary care practice by implementing decision aids. N C Med J. 2009;70(2): 136-9.

19. Gaseem A, Barry MJ, Denberg TD, Owens DK, Shekelle P. Screening for prostate cancer: a guidance statement from the Clinical Guidelines Committee of the American College of Physicians. Ann Intern Med. 2013;158(10):761-9.

20. Smith RA, Cokkinides V, Brooks D, Saslow D, Brawley OW. Cancer Screening in the United States, 2010: A Review of Current American Cancer Society Guidelines and Issues in Cancer Screening. CA: a cancer journal for clinicians. 2010;60(2):99-119.

21. Moyer VA. Screening for Prostate Cancer: U.S. Preventive Services Task Force Recommendation Statement. Ann Intern Med. 2012;157(2):120-34.

22. Partin MR, Nelson D, Radosevich D, Nugent S, Flood AB, Dillon N, et al. Randomized trial examining the effect of two prostate cancer screening educational interventions on patient knowledge, preferences, and behaviors. J Gen Intern Med. 2004;19(8):835-42.

23. Elwyn G, Lloyd A, Joseph-Williams N, Cording E, Thomson R, Durand MA, et al. Option Grids: shared decision making made easier. Patient Educ Counsel. 2013;90(2):207-12. 
24. Glasgow RE, Lichtenstein E, Marcus AC. Why don't we see more translation of health promotion research to practice? Rethinking the efficacy-to-effectiveness transition. Am J Publ Health. 2003;93(8): 1261-7.
25. Wolf AMD, Wender RC, Etzioni RB, Thompson IM, D'Amico AV, Volk RJ, et al. American Cancer Society Guideline for the Early Detection of Prostate Cancer: Update 2010. CA: a cancer journal for clinicians. 2010;60(2):70-98. 